\title{
Melatonin in der topischen Behandlung der androgenetischen Alopezie
}

\author{
Topical Melatonin for Treatment of Androgenetic Alopecia
}

Autoren

Institute
T. W. Fischer ${ }^{1}$, R. M. Trüeb ${ }^{2}$, G. Hänggi ${ }^{3}$, M. Innocenti ${ }^{4}$, P. Elsner $^{5}$

Klinik für Dermatologie, Allergologie und Venerologie, Universität zu Lübeck

Dermatologische Praxis und Haarcenter Professor Trüeb, Zentrum Wallisellen, Wallisellen/Zürich, Schweiz

Gabriella Hänggi, Thalwil, Schweiz

${ }^{4}$ IDE Istituto Dermatologico Europeo, Mailand/Italien

Klinik für Hautkrankheiten, Universitätsklinikum Jena

\section{Bibliografie}

DOI http://dx.doi.org/

10.1055/s-0030-1256701

Online-Publikation: 8. 8. 2011

Akt Dermatol 2010; 36:

410-418 ๑ Georg Thieme

Verlag KG Stuttgart · New York ISSN 0340-2541

\section{Korrespondenzadresse}

Priv.-Doz. Dr. med.

Tobias W. Fischer

Klinik für Dermatologie, Allergologie und Venerologie Universität zu Lübeck

Ratzeburger Allee 160

23538 Lübeck

Tobias.Fischer@uk-sh.de

\section{Zusammenfassung \\ $\nabla$}

Auf der Suche nach alternativen Substanzen zu Finasterid und Minoxidil für die Behandlung der androgenetischen Alopezie (AGA) wurde Melatonin, ein starkes Antioxidanz und Wachstumsmodulator, als Kandidat aus In-vitro- und In-vivoUntersuchungen identifiziert. Insgesamt fünf klinische Studien zeigten bei guter Verträglichkeit positive Effekte einer topischen Melatonin-Behandlung auf die AGA bei Männern und Frauen: (1) Pharmakodynamisch zeigte sich unter einmal abendlicher topischer Anwendung kein signifikanter Einfluss auf die endogenen MelatoninPlasmaspiegel. (2) In einer Anwendungsbeobachtung mit 30 Männern und Frauen mit Prüfarztund Probandenfragebögen zeigte sich nach 30 und 90 Tagen ein signifikanter Rückgang des Schweregrades der Alopezie $(p<0,001)$. (3) Mittels digitaler, Software-gestützter Auflichtmikroskopie (TrichoScan) wurde bei 35 Männern mit

\section{Einleitung}

\section{$\nabla$}

Das Kopfhaar stellt für Männer wie für Frauen ein Attribut für körperliche Attraktivität und Jugendlichkeit dar, weshalb Verlust des Kopfhaares das Selbstwertgefühl und die Lebensqualität Betroffener signifikant beeinträchtigen kann $[1,2]$. Die weitaus häufigste Ursache von Haarverlust bei Mann und Frau ist die androgenetische Alopezie (AGA), bei der es sich um einen genetisch determinierten, altersabhängig fortschreitenden Haarverlust mit geschlechtsspezifischen Unterschieden in der Häufigkeit und Ausprägung handelt $[2,3]$. Die Prävalenz der AGA bei Männern im Alter von 18 bis 29 Jahren liegt bei ca. $12 \%$ und bei 40- bis 49-Jährigen bei ca. 50\% [4]. Bei den Frauen liegt eine AGA bei ca. $17 \%$ der 30- bis 49-Jährigen vor, wobei die Prävalenz nach der Menopause stark zunimmt und bei den 50 bis 69-Jährigen
AGA bei 54,8 bzw. 58,1\% der Patienten nach 3 und 6 Monaten eine signifikante Zunahme der Haardichte um $29 \%$ bzw. $41 \%$ gemessen (M0:

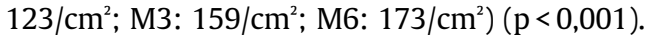
(4) Bei 60 Männern und Frauen mit Haarausfall wurde eine signifikante Reduktion des Haarverlustes bei den Frauen festgestellt, während bei den Männern der Haarausfall konstant blieb $(p<0,001)$. (5) In einer Multizenterstudie mit über 1800 Probanden in 200 Zentren über 3 Monate nahm der Anteil der Patienten mit 2- bis 3fach positivem Pull-Test von $61,6 \%$ auf $7,8 \%$ ab und der Anteil der Patienten mit negativem PullTest von $12,2 \%$ auf $61,5 \% \mathrm{zu}(\mathrm{p}<0,001)$. Zusätzlich wurde ein Rückgang der Seborrhö und der seborrhoischen Dermatitis der Kopfhaut beobachtet. Da die Sicherheit und Verträglichkeit in allen Studien gut war, kann die topische Anwendung einer kosmetischen Melatonin-Lösung eine sinnvolle Behandlungsoption bzw. Ergänzung bei androgenetischer Alopezie darstellen.
$23 \%$ bis $25 \%$ betragen kann [4]. Die AGA beginnt bei Männern gewöhnlich mit Ausbildung von Geheimratsecken und schreitet fort, indem die Stirnhaargrenze zurückweicht und eine Wirbelglatze entsteht. Im weiteren Verlauf können sich die kahlen Stellen bis zur Bildung einer Vollglatze vergrößern, bei der lediglich ein Haarkranz am Hinterkopf und an den Schläfen verbleibt $[5,6]$. Bei Frauen zeigt die AGA in der Regel eine langsamere Progression als bei Männern und geht mit einer allmählichen, diffusen Ausdünnung der Haare im Oberkopfbereich unter Beibehaltung der frontalen Haarlinie einher [7].

Bisher sind nur zwei FDA- und EMEA-registrierte Therapeutika zur Behandlung der AGA zugelassen, systemisches Finasterid (nur Männer) und topisches Minoxidil (Frauen und Männer) [4]. Da deren Wirkung ihre Grenzen aufweist und unerwünschte Wirkungen, die zum Absetzen der The- 
rapie zwingen, bei beiden Wirkstoffen vorkommen können (z. B. Gynäkomastie bei Finasterid [8] oder Hypertrichose bei Minoxidil [9]), erscheint es sinnvoll, alternative Wirkstoffe zur Behandlung der AGA zu identifizieren.

Nach dem derzeitigen pathogenetischen Verständnis liegt der AGA eine genetisch bedingte Hypersensitivität der Haarfollikel auf Androgene zugrunde. Bei Männern spielt vor allem die erhöhte Aktivität des Enzyms 5-alpha-Reduktase Typ II in genetisch prädisponierten Haarfollikeln der Temporal- und Vertex-Region eine wichtige Rolle, welche das im Blut zirkulierende testikuläre Testosteron im Haarfollikel zu Dihydrotestosteron (DHT) metabolisiert [2,3]. Bei Frauen ist eher die verminderte Aktivität der Aromatase bedeutsam, welche das im Blut zirkulierende ovariale Testosteron zu 17-beta-Östradiol umwandelt [3]. Durch Anstieg der lokalen Konzentration von DHT mit Bindung an die verstärkt exprimierten Androgen-Rezeptoren in prädisponierten Haarfollikeln $[2,4]$ entwickelt sich eine progrediente Verkürzung der Anagenphase zugunsten verlängerter Telogenphasen, welche mit einer progressiven Miniaturisierung der Haarfollikel einhergeht. Als Folge ergibt sich eine allmähliche Umwandlung von dicken, pigmentierten Terminalhaaren zu feinen, unpigmentieren Vellushaaren (miniaturisierte Haare) [10].

Neben den Androgen-abhängigen Veränderungen der Morphologie und Wachstumsdynamik der Haarfollikel scheint bei der Pathogenese der AGA auch eine follikuläre Mikroentzündung und Fibrose eine Rolle zu spielen [11], welche möglicherweise durch die mikrobielle Residentflora bei Seborrhö, Toxine und oxidativen Stress ausgelöst wird [12]. Darüber hinaus wirken auch Alterungsprozesses und andere Faktoren wie Ultraviolettstrahlung $[13,14]$, Rauchen $[15,16]$ und Umweltschadstoffe [17] negativ auf die Haarfollikel ein. Diese belastenden Einflüsse führen zur Bildung von freien Radikalen, welche durch die zelleigenen Schutzmechanismen nicht mehr ausreichend abgebaut werden können und deshalb die Haarfollikel schädigen $[10,12]$. Kürzlich wurde nachgewiesen, dass die Papillenfibroblasten bei AGA eine erhöhte Empfindlichkeit auf oxidativen Stress aufweisen [18].

Melatonin (N-Acetyl-5-methoxy-tryptamin) ist ein möglicher Kandidat, der dem beschriebenen oxidativen Stress bei Haarausfall, allgemein aber auch bei der AGA entgegenwirken kann, da starke antioxidative Eigenschaften für diese Substanz beschrieben wurden [19]. Ursprünglich als von der Glandula pinealis in zirkadianer Rhythmik gebildetes und freigesetztes Neurohormon entdeckt [20], regelt Melatonin eine Vielzahl von physiologischen Vorgängen wie saisonale Biorhythmen, den täglichen SchlafWach-Rhythmus und beeinflusst den Alterungsprozess [21-23]. Durch seine Eigenschaften als starkes Antioxidanz und aktiver Fänger von freien Radikalen [19] übt Melatonin aber v.a. protektive und antiapoptotische Wirkungen aus, welche die funktionelle Integrität von nicht-neoplastischen Zellen gewährleisten können [24-28].

Nach neueren Erkenntnissen sind zahlreiche periphere Organe nicht nur Ziel von Melatonin-Bioaktivität, sondern gleichzeitig auch Ort für extrapineale Melatonin-Synthese, Regulation und Metabolismus von Melatonin. In der menschlichen Haut konnte ein melatoninerges Enzymsystem nachgewiesen werden, das die für die Melatonin-Biosynthese benötigten spezifischen Enzyme vollständig exprimiert [29]. Zusätzlich weisen Keratinozyten, Melanozyten und Fibroblasten funktionelle Melatonin-Rezeptoren auf, welche an phänotypischen Wirkungen wie der zellulären Proliferation und Differenzierung beteiligt sind [30]. Als Schutz vor UV-induzierten Schäden wurde ein wirksames melatoninerges antioxidatives System der Haut identifiziert [31].
Wie in der Haut konnte auch in humanen Haarfollikeln sowohl die Synthese von Melatonin als auch die Expression von Melatonin-Rezeptoren nachgewiesen und ein Einfluss auf die Haarwachstumszyklen beobachtet werden [32-34]. Melatonin führt bei in-vitro-kultivierten humanen Anagen-Haarfollikeln in einer Konzentration von $30 \mu \mathrm{M}$ im Vergleich zum Kulturmedium alleine oder auch zu deutlich höheren Konzentrationen im MillimolBereich (1-5 mM) zu einem signifikant schnelleren Wachstum der Haarfollikel [35]. Da die Stimulation der Haarfollikel durch den potenten Melatonin-Antagonisten Phenyl-2-propionaminotetralin unterdrückt werden kann, erscheint ein Rezeptor-vermittelter Wirkmechanismus durch Melatonin sehr wahrscheinlich [35]. Dass Melatonin-Rezeptoren im Verlauf der Haarzyklusphasen reguliert werden, konnte zumindest im Mausmodell bereits gezeigt werden [34]. Diese präklinischen Daten der Melatonin-vermittelten Haarwachstumsstimulation werden durch die Resultate einer doppelblinden, placebo-kontrollierten Pilotstudie unterstützt, in welcher die topische Behandlung mit $1 \mathrm{ml}$ einer 0,1-\%igen alkoholischen Melatonin-Lösung bei Frauen mit AGA und mit diffuser Alopezie nach sechs Monaten gegenüber Placebo einen signifikanten Anstieg der nachweisbaren Anagenhaare im Okzipital- bzw. Frontal-Bereich bewirkte [36].

\section{Material und Methoden \\ $\nabla$}

Entwicklung einer pharmakologischen Formulierung Aufgrund der positiven Wirkungen von Melatonin auf das Haarwachstum wurde von der Firma ASATONA AG (Zug, Schweiz) eine topisch anwendbare, kosmetische Haarlösung mit einem Melatoningehalt von $0,0033 \%$ entwickelt, mit der Zielsetzung, den Alterungsprozess des Haares zu verlangsamen sowie als adjuvante Behandlung der AGA zu fungieren. Zusätzlich zu Melatonin wurde für diese Formulierung Ginkgo biloba zugegeben, das durch die Verbesserung der Ernährung in den Haarwurzeln das Haarwachstum günstig beeinflusst und ebenfalls ausgeprägte antioxidative Eigenschaften besitzt, sowie Biotin, welches einen wichtigen Mikro-Nährstoff zur Unterstützung der Zellfunktionen darstellt.

\section{Studiendurchführung}

Im Zeitraum von Januar 2003 bis Oktober 2006 wurden im Auftrag der Firma ASATONA AG fünf Studien zu der oben genannten kosmetischen Melatonin-Lösung durchgeführt, wovon die erste Studie die Pharmakokinetik der Melatonin-Lösung untersuchte und die weiteren 4 Studien in verschiedenen Probanden-Kollektiven anhand verschiedener Untersuchungsparameter die Wirkung der Melatonin-Lösung auf Haarausfall evaluierten. Die Untersuchungsparameter und Studiendesigns sind der besseren Zuordnung wegen jeweils bei den Ergebnissen der einzelnen Studien genannt. Die Studien wurden wie folgt benannt: MEL-COS-1 (Studie 1), MEL-COS-AS01 (Studie 2), MEL-COS-AS03 (Studie 3), MEL-COS-AS04 (Studie 4) und MEL-COS-AS05 (Studie 5).

\section{Ergebnisse \\ $\nabla$}

Pharmakokinetik der kosmetischen Melatonin-Lösung (Studie 1; MEL-COS-1)

Zur Beurteilung der biologischen und klinischen Verträglichkeit der kosmetischen Melatonin-Lösung $(0,0033 \%)$ wurden in einer doppelblinden, placebo-kontrollierten Crossover-Studie bei 4 fertilen und 4 postmenopausalen gesunden Frauen die pharmakoki- 


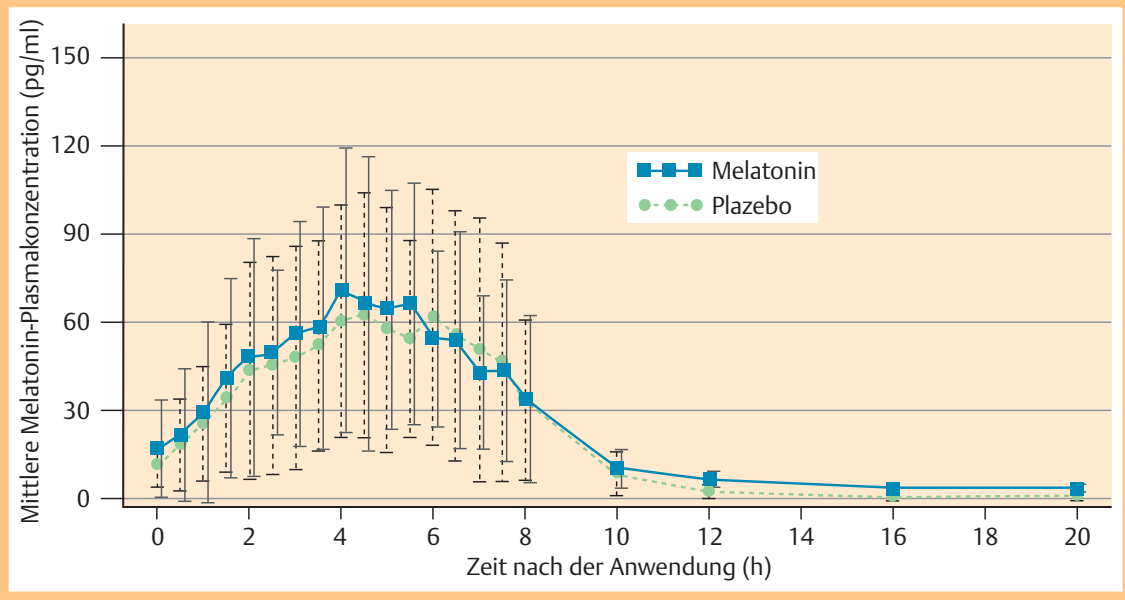

Abb. 1 Mittlere Plasmakonzentration nach der zweiwöchigen Behandlung mit der kosmetischen Melatonin-Lösung.

netischen Parameter der kosmetischen Haarlösung, die einmal täglich vor dem Zubettgehen auf die Kopfhaut aufgetragen wurde, ermittelt [37]. Die Studie wurde von 1/2003 bis 4/2003 im „Forenap Centre Hospitalier“ in Rouffach, Frankreich, nach Begutachtung und positivem Ethik-Votum durchgeführt. Die Rekrutierung der Probanden erfolgte nach mündlicher und schriftlicher Auklärung und Einverständniserklärung. Zur Bestimmung der Pharmakokinetik von Melatonin wurde am Tag 14 der Testperiode über 8 Std. nach Applikation in 30-minütigem Abstand und 10, 12, 16 und 20 Std. nach Applikation Blut abgenommen. Die Analyse der Blutproben ergab, dass die wiederholte Anwendung der kosmetischen Melatonin-Lösung keinen negativen Einfluss im Sinne einer Anhebung oder Suppression der physiologischen endogenen Melatonin-Sekretion hatte. Der mittlere MelatoninPlasmaspiegel verlief über 20 Stunden ähnlich wie nach der Anwendung von Placebo, die maximale Melatonin-Plasmakonzentration in der Melatonin-Gruppe lag etwas höher als in der Placebo-Gruppe $(83,4 \mathrm{pg} / \mathrm{ml}$ vs. $71,2 \mathrm{pg} / \mathrm{ml})$ und wurde etwas früher erreicht $(\bullet$ Abb. 1).

Es konnte beobachtet werden, dass die mittlere Melatonin-Plasmakonzentration bei den fertilen Frauen deutlich höher war als bei den postmenopausalen Frauen. Die kumulative Menge des innerhalb von 24 Stunden im Urin ausgeschiedenen 6-Hydroxymelatoninsulfates, der wichtigste Metabolit von Melatonin und Melatonin-Messäquivalent, war in beiden Gruppen vergleichbar, wobei sich in beiden Gruppen auch eine hohe inter-individuelle Schwankungsbreite der Werte fand. Es konnten keine Veränderungen der Vitalparameter (Blutdruck, Herzfrequenz) und des Elektrokardiogramms unter Melatonin-Behandlung festgestellt werden. Die Resultate der beiden nach Abschluss der zweiwöchigen Behandlung durchgeführten neurokognitiven Tests „Critical Flicker Fusion“ und „Multiple Choice Reaction Time“ ergaben, dass die wiederholte Anwendung der kosmetischen MelatoninLösung im Vergleich zur Placebo-Lösung keinen signifikanten Einfluss auf die Reaktionszeit und auf die kortikalen Arousals hatte.

Die Behandlung mit der kosmetischen Melatonin-Lösung erwies sich als gut verträglich, da im Vergleich zu Placebo weder signifikante Veränderungen der verschiedenen Labor- und Kreislaufparameter noch Auswirkungen auf das Zentralnervensystem festgestellt wurden. Im Verlauf der Studie wurden nur wenige unerwünschte Wirkungen wie mittelschwere Kopfschmerzen und gastrointestinale Störungen beobachtet, wobei deren Inzidenz in der Melatonin-Gruppe und der Placebo-Gruppe identisch war und kein kausaler Zusammenhang mit der Anwendung der Studienmedikation festgestellt wurde.

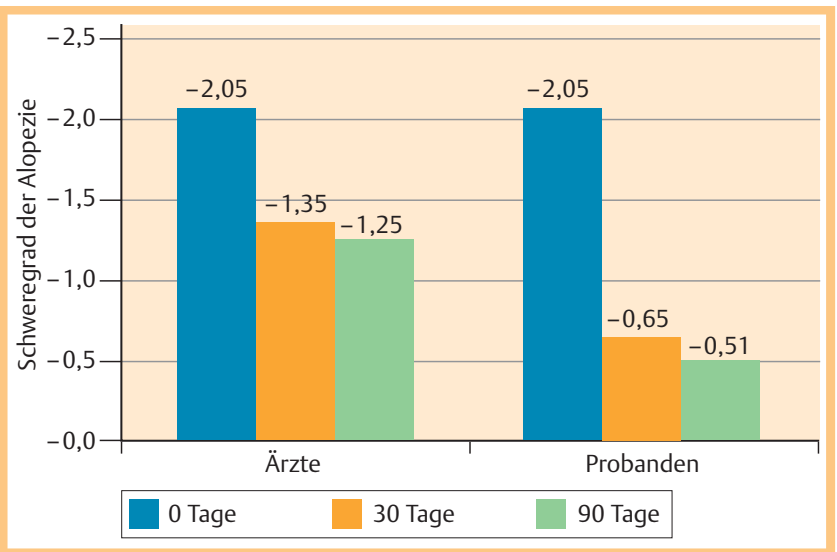

Abb. 2 Objektive und subjektive Beurteilung des Schweregrads der Alopezie.

\section{Wirkung bei der AGA im frühen Stadium}

(Studie 2; MEL-COS-AS01)

Nach der Klärung der Sicherheitsaspekte der kosmetischen Melatonin-Lösung in der pharmakokinetischen Studie (Studie 1; MELCOS-1) wurde in einer offenen Anwendungsbeobachtung bei 15 Frauen mit AGA im Stadium I bis II nach Ludwig [7] und bei 15 Männern mit einer AGA im Stadium I bis II nach Hamilton/Norwood [5,6] im Alter von 18-40 Jahren die Wirksamkeit der jeweils abends angewendeten kosmetischen Melatonin-Haarlösung untersucht [38]. Die Studie wurde von 3/2003 bis 6/2003 im „Instituto Dermatologico Europeo“ (IDE) in Mailand, Italien, nach positiver Ethik-Begutachtung nach GCP durchgeführt. Vor Teilnahme an der Studie gaben die Probanden ihre schriftliche Einverständniserklärung. Mittels objektiver Prüfarzt-Untersuchungsbögen und subjektiver Patientenfragebögen wurde bereits nach 30 Tagen ein signifikanter Rückgang des Schweregrades der Alopezie festgestellt, der nach 90 Tagen nochmals signifikant verstärkt wurde ( $<<0,001)$ ( $\bullet$ Abb. 2 ).

Die kosmetischen Eigenschaften „Duft“, „Konsistenz“ und „Absorption“ wurden mit 2,67, 2,52 bzw. 2,57 von maximal 3 Punkten bewertet, während der fettende Einfluss auf die Haare mit 0,43 und die Kämmbarkeit mit 0,76 von 1 Punkt beurteilt wurde. Die Zufriedenheit mit dem Produkt wurde mit 1,80 von 2 Punkten angegeben, und die Unverträglichkeit wurde nach 30 Tagen mit 0,54 und nach 90 Tagen mit 0,52 von maximal 3 Punkten bewertet. 
TrichoScan-Untersuchung zur Beurteilung der Wirksamkeit (Studie 3; MEL-COS-AS03)

Im Hinblick auf eine zuverlässige und technisch-methodisch objektive Beurteilung des therapeutischen Nutzens der kosmetischen Melatonin-Lösung wurde in einer weiteren offenen, klinisch-kontrollierten Studie die Wirksamkeit und Verträglichkeit der Melatonin-Haarlösung, die von 35 Männern (Alter: 18-41 Jahre) mit AGA im Stadium I oder II nach Hamilton/Norwood während 6 Monaten jeweils am Abend auf die Kopfhaut aufgetragen wurde, mit Hilfe der TrichoScan-Methode ermittelt [39]. Die Studie wurde von 4/2004 bis 4/2005 im „Instituto Dermatologico Europeo" (IDE) in Mailand, Italien, nach positiver Ethik-Begutachtung nach GCP durchgeführt. Vor Teilnahme an der Studie gaben die Probanden ihre schriftliche Einverständniserklärung. Die TrichoScan-Methode ist eine auflichtmikroskopisch und Software-gestützte Messmethode zur Messung von Haarzahl (Anzahl Haare $/ 0,7 \mathrm{~cm}^{2}$ ), Haardichte (Anzahl Haare $/ 1 \mathrm{~cm}$ ), des Haardurchmessers, der Anagen-/Telogen-Rate und der Vellus-/Terminalhaar-Rate $[40,41]$. Die Resultate dieser Studie zeigten nach 3 und 6 Monaten jeweils bei 54,8\% der Teilnehmer eine Zunahme der Haarzahl (Anzahl Haare/0,7 $\mathrm{cm}^{2}$ ), und bei $54,8 \%$ bzw. 58,1\% der Teilnehmer wurde nach 3 bzw. 6 Monaten eine Verbesserung der Haardichte (Anzahl Haare/1 $\mathrm{cm}^{2}$ ) verzeichnet.

Die Zunahme der Haarzahl lag bei 29,2\% (3 Monate vs. 0 Monate) und $42,7 \%$ (6 Monate vs. 0 Monate) mit jeweils statistischer Signifikanz $(\mathrm{p}<0,001)$ (Monat 0: 85,76 $\pm 27,0$; Monat 3: 110,82 $\pm 31,7$; Monat 6: $122,35 \pm 40,5$ ). Bei der Haardichte konnte eine Zunahme um 29,1\% und 40,9\% nach 3 und 6 Monaten verzeichnet werden (Monat 0: $123,15 \pm 39,0$; Monat 3: $159,03 \pm 46,8$; Monat 6: $173,56 \pm 58)$. Die Werte der Haardichte unterschieden sich ebenfalls signifikant $(p<0,001)$.

$\mathrm{Zu}$ den verschiedenen Visiten wurde nach der objektiven Prüfarzt-Beurteilung unter der Behandlung mit der kosmetischen Melatonin-Lösung bei 26,6\% (Tag 30), 48,2\% (Tag 90) und 32,1\% (Tag 180) der Probanden eine Verbesserung des Haarverlustes festgestellt, wobei dieser Anteil nach 90 Tagen am höchsten war und sogar einen geringen Anteil von Probanden mit einem erneuten Haarwachstum umfasste. Darüber hinaus reduzierte sich vom Zeitpunkt 30 Tage bis zum Zeitpunkt 90 Tage der Anteil der Probanden mit unverändertem Haarverlust von rund $73,3 \%$ auf $48,2 \%$ und blieb auch auf diesem Wert bis zum Tag 180 . Bei einem kleinen Anteil von 3,4\% (90 Tage) und 19,3\% (180 Tage) setzte sich der Haarverlust fort ( $\bullet$ Abb. 3).

Nach einer Behandlungsdauer von 30, 90 und 180 Tagen waren gemäß der subjektiven Probandenfragebögen jeweils 30\%, 34,5\% und $29 \%$ der Probanden mit der Behandlung zufrieden und $70 \%$, $58,6 \%$ und 58,0\% weitgehend zufrieden, wohingegen der Anteil der mit der Behandlung nicht zufriedenen Probanden im Verlauf der Studie mit 0\% (30 Tage), 6,8\% (90 Tage) und 12,9\% (180 Tage) relativ gering war. Zu den kosmetischen Eigenschaften wurde von den Probanden ein Fragebogen beantwortet, der die Charakteristika Duft, Konsistenz, Absorption, Öligkeit und Kämmbarkeit nach Anwendung des Produktes umfasste.

Der Duft wurde von 83,8\% der Probanden als gut und von 6,4\% als hervorragend beschrieben, während 9,6\% mit dem Duft nicht zufrieden waren. Die Konsistenz wurde von $77,4 \%$ als gut, von $9,6 \%$ als exzellent und von $12,9 \%$ als weniger gut beschrieben. Die Absorption des Produktes auf der Kopfhaut wurde von 80,6\% als gut, von $12,9 \%$ als sehr gut und von $6,4 \%$ als schlecht empfunden. Von 93\% wurde keine Öligkeit der Kopfhaut nach Applikation beobachtet und die Kämmbarkeit wurde von $96,7 \%$ als gut eingestuft. Ein einziger Proband berichtete über gelegentlichen

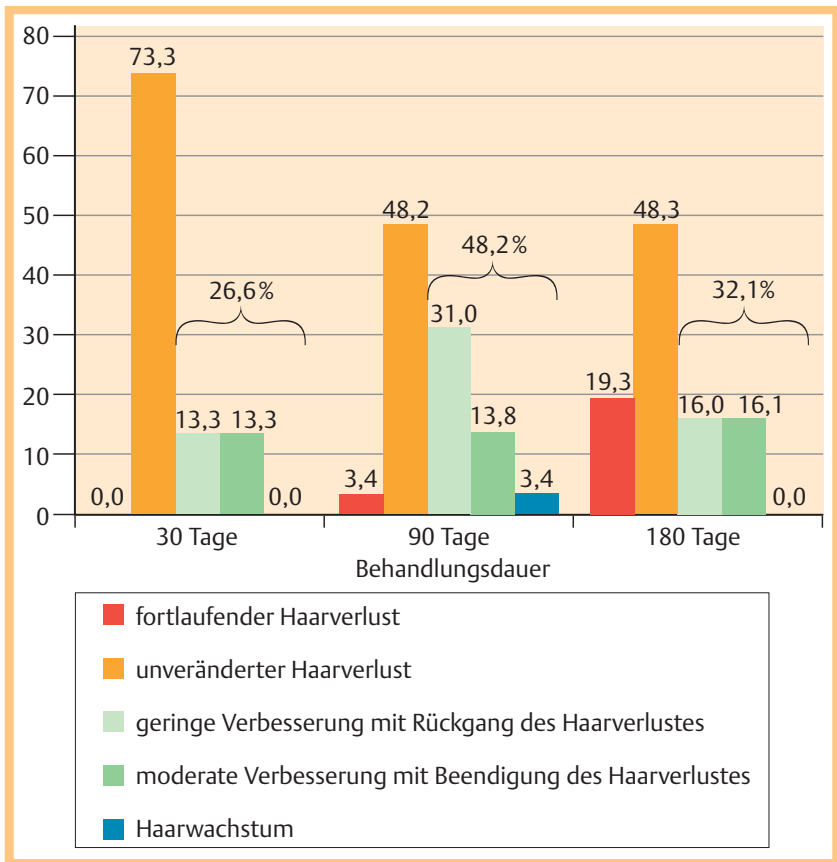

Abb. 3 Rückgang des Haarverlustes, beurteilt durch objektive PrüfarztUntersuchungsbögen. Prozentwert über Klammer: Zusammenfassung der Prozentwerte von „geringe Verbesserung mit Rückgang des Haarverlustes“, „moderate Verbesserung mit Beendigung des Haarverlustes“ und „Haarwachstum“.

Juckreiz nach der Anwendung des Produktes. Insgesamt wurde die kosmetische Melatonin-Haarlösung von $93,5 \%$ als gut (80,6\%) bis sehr gut (12,9\%) eingestuft.

Kammtest in Coiffeur-Salons (Studie 4; MEL-COS-AS04) Im Rahmen einer offenen Anwendungsbeobachtung wurde die Wirksamkeit der kosmetischen Melatonin-Lösung in vier Coiffeur-Salons bei 40 männlichen und 20 weiblichen Probanden (mittleres Alter 41 Jahre) mit Frühstadien von Haarausfall oder Haarlichtung untersucht, welche die Melatonin-Haarlösung während 90 Tagen jeweils am Abend anwendeten [42]. Die Studie wurde als offene Studie von 6/2005 bis 9/2005 in 4 Coiffeur-Salons in Tampa, FL, USA, als „Non-FDA-related cosmetic study“ durchgeführt. Vor Teilnahme an der Studie gaben die Probanden ihre schriftliche Einverständniserklärung. Gemäß der Einschätzung der Coiffeure auf einer 4-Punkte-Skala trat unter der Behandlung mit der kosmetischen Melatonin-Haarlösung eine Verbesserung der Haartextur ein, welche sowohl in der Gruppe der weiblichen Probanden als auch in der Gruppe der männlichen Probanden statistische Signifikanz erreichte $(p=0,002$ bzw. $p=8 \times 10^{-5}$ ). Darüber hinaus stellten die Coiffeure eine Reduktion des Haarverlustes fest, die ebenfalls bei den Frauen und den Männern statistisch signifikant war $\left(\mathrm{p}=4 \times 10^{-6}\right.$ bzw. $\left.\mathrm{p}=3 \times 10^{-5}\right)$ (๑ Tab. 1).

Der durch die Probanden beim morgendlichen Kämmen mit Hilfe des 60-Sekunden-Haarzähltests [43] bestimmte mittlere Haarverlust nahm bei den Frauen während der ersten 40 Tage stark und während der folgenden 40 Tagen nochmals signifikant weiter ab, wogegen bei den Männern der mittlere Haarverlust im Verlauf der Studie nahezu konstant blieb, aber wesentlich geringer war als bei den Frauen. Bezüglich der Zufriedenheit mit der Behandlung zeigte sich in der Gesamtgruppe ein Trend zu einer Verbesserung, wobei die Zufriedenheit in der Gruppe der weibli- 
Tab. 1 Verbesserung des Haarzustandes.

\begin{tabular}{|c|c|c|c|}
\hline & $\begin{array}{l}\text { Gesamt- } \\
\text { gruppe }\end{array}$ & Frauen & Männer \\
\hline $\begin{array}{l}\text { Haartextur } \\
\text { (4-Punkte- } \\
\text { Skala) }\end{array}$ & $\begin{array}{l}0,74 \\
\left(p=7 \times 10^{-9}\right)\end{array}$ & $\begin{array}{l}1,08 \\
(p=0,002)\end{array}$ & $\begin{array}{l}0,58 \\
\left(p=8 \times 10^{-5}\right)\end{array}$ \\
\hline $\begin{array}{l}\text { Haarverlust } \\
\text { (5-Punkte- } \\
\text { Skala) }\end{array}$ & $\begin{array}{l}0,59 \\
\left(p=1 \times 10^{-7}\right)\end{array}$ & $\begin{array}{l}0.69 \\
\left(p=4 \times 10^{-6}\right)\end{array}$ & $\begin{array}{l}0,55 \\
\left(p=3 \times 10^{-5}\right)\end{array}$ \\
\hline $\begin{array}{l}\text { Zufriedenheit } \\
\text { (4-Punkte- } \\
\text { Skala) }\end{array}$ & $\begin{array}{l}0,22 \\
(p=0,045)\end{array}$ & $\begin{array}{l}0.46 \\
(p=0,002)\end{array}$ & $\begin{array}{l}0,10 \\
(p=0,50)\end{array}$ \\
\hline
\end{tabular}

chen Probanden signifikant zunahm $(p=0,002)$ und in der Gruppe der männlichen Probanden nahezu unverändert blieb ( Tab. 1). Im Verlauf der Studie traten bei 4 Probanden leichte Nebenwirkungen auf, bei denen es sich um eine vorübergehende Rötung, Empfindlichkeit, Juckreiz oder Brennen handelte. Diese führten jedoch nicht zu einem Ausscheiden aus der Studie.

\section{Multizenterstudie belegt Wirksamkeit \\ (Studie 5; MEL-COS-ASO5)}

Zur umfassenderen Beurteilung des therapeutischen Nutzens der kosmetischen Melatonin-Lösung wurde in 200 dermatologischen Zentren oder Praxen eine große, offene Multizenterstudie durchgeführt, in welcher 901 Männer (47,6\%) mit einer AGA im Stadium I oder II nach Hamilton und 990 Frauen (52,4\%) mit einer AGA im Stadium I oder II nach Ludwig während 90 Tagen jeweils abends mit einer Melatonin-haltigen, kosmetischen Haarlösung behandelt wurden [44]. Die Studie wurde von 3/2004 bis 10/ 2006 vom „Instituto Dermatologico Europeo (IDE) in Mailand, Italien, nach positiver Ethik-Begutachtung nach GCP geleitet. Vor Teilnahme an der Studie gaben die Probanden ihre schriftliche Einverständniserklärung.

Das klinische Ansprechen wurde mittels Pull-Test evaluiert, ein semi-quantitativer Test zur Untersuchung der Haarausfall-Aktivität $[4,45]$. Ein positiver Pull-Test wird in drei Stufen angegeben $(+,++,+++)$ oder als negativ (-) beurteilt. Der durch die Ärzte an den Tagen 30 und 90 vorgenommene Pull-Test ergab, dass die Anteile der Probanden mit schwerem und mittelschwerem Haarverlust im Verlauf der Studie signifikant von 61,6\% auf 33,7\% (30 Tage) und 7,8\% (90 Tage) abnahmen ( $p<0,001)$. Darüber hinaus stieg der Anteil der Probanden, bei denen kein Haarverlust nachgewiesen wurde, unter der Therapie mit der kosmetischen Melatonin-Lösung signifikant von $12,2 \%$ auf $25,5 \%$ (30 Tage) und $61,5 \%$ (90 Tage) an ( $\mathrm{p}<0,001$; Student's t-Test) ( $\bullet$ Abb. 4).

Nach der objektiven Prüfarzt-Beurteilung hatte die Behandlung mit der kosmetischen Melatonin-Lösung nach 30 und 90 Tagen bei 59,4\% bzw. 66,4\% der Probanden zu einer signifikanten Verbesserung des Haarverlustes geführt $(\mathrm{p}<0,001)$, während bei 4,5\% bzw. 22,5\% der Probanden sogar ein erneutes Wachstum der Haare verzeichnet wurde $(\mathrm{p}<0,001)(\bullet$ Abb. 5).

Gemäß der von den Probanden vorgenommenen subjektiven Selbsteinschätzung stieg der Anteil der Probanden, die an leichtem oder schwerem Haarverlust litten, nach 30 Tagen von $71,4 \%$ auf $82,0 \%$ zunächst an und nahm dann aber nach 90 Tagen deutlich auf 60,0\% ab. Dementsprechend sank der Anteil der Probanden, die keinen Haarverlust beklagten, nach 30 Tagen von 27,7\% auf $18 \%$ und stieg anschließend aber nach 90 Tagen deutlich auf $40 \%$ an $(\bullet$ Abb. 6$)$.

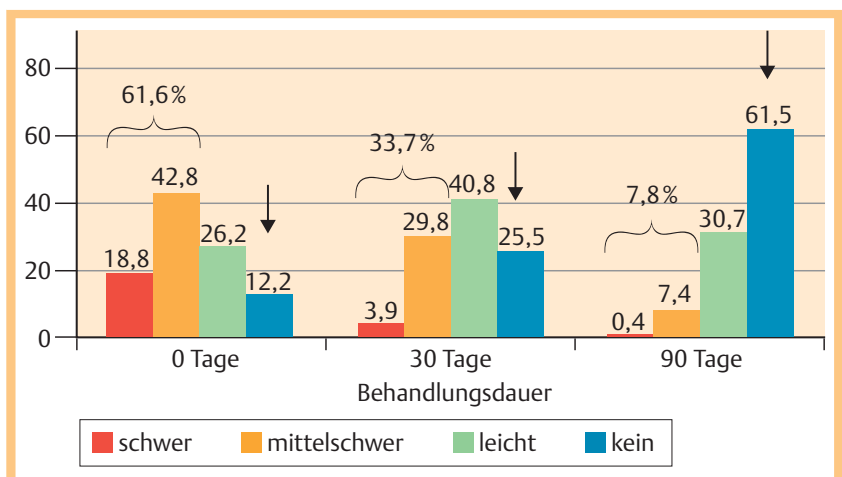

Abb. 4 Rückgang des Haarverlustes durch Pull-Test. Unterschied gegenüber Anfangswert: $p<0,001$ bei 30 und 90 Tagen. Prozentwert über Klammer: schwerer bis mittelschwerer Haarverlust; vertikale Pfeile: kein Haarverlust.

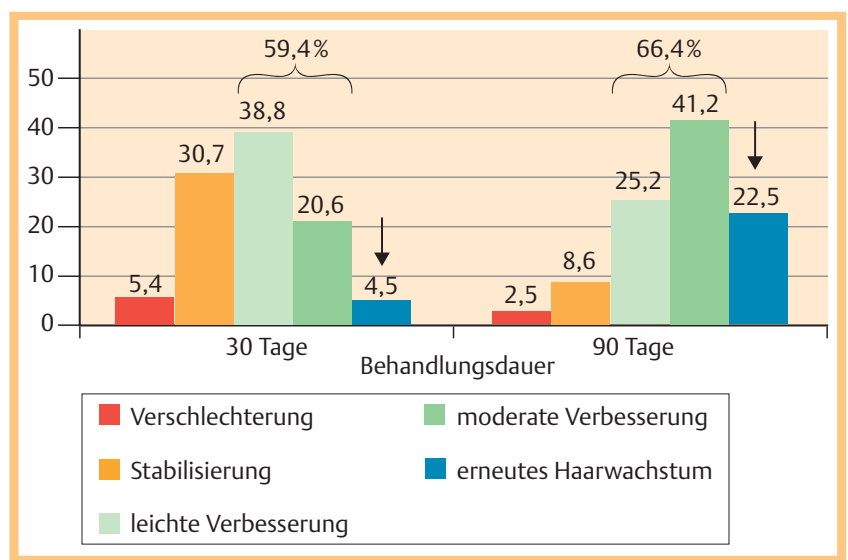

Abb. 5 Beurteilung der Wirksamkeit durch die Ärzte. Unterschied gegenüber Anfangswert: $p<0,001$ bei 30 und 90 Tagen. Prozentwert über Klammer: leichte bis moderate Verbesserung; vertikale Pfeile: erneutes Haarwachstum

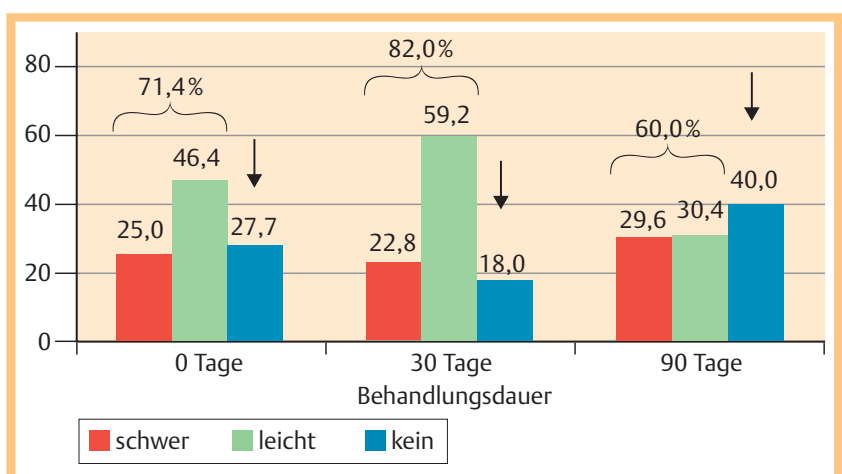

Abb. 6 Einschätzung der Probanden. Unterschied gegenüber Anfangswert: $p<0,001$ bei 30 und 90 Tagen. Prozentwert über Klammer: leichter bis schwerer Haarverlust; vertikale Pfeile: kein Haarverlust.

Zusätzlich zum Rückgang des Haarverlustes bewirkte die Behandlung mit der Melatonin-Lösung eine Verbesserung der Seborrhö. Der Anteil der Probanden mit einer mittelschweren oder schweren Seborrhö von anfänglich 35,7\% wurde nach 30 und 90 Tagen auf $18 \%$ bzw. 5,4\% reduziert. Entsprechend nahm der Anteil der Probanden, der keine Seborrhö zeigte, von 32,5\% (Baseline) auf 45,4\% (30 Tage) und 67,6\% (90 Tage) signifikant $\mathrm{zu}$ $(\mathrm{p}<0,001)(\bullet$ Abb. 7). 


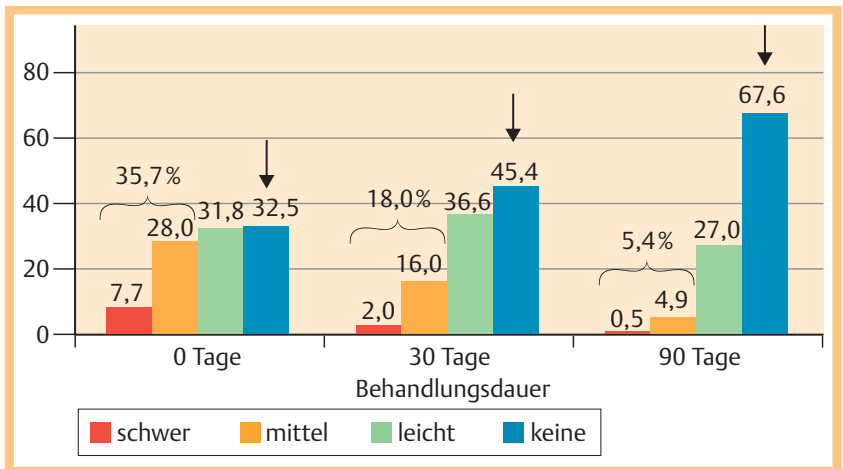

Abb. 7 Rückgang der Seborrhö. Unterschied gegenüber Anfangswert: $\mathrm{p}<0,001$ bei 30 und 90 Tagen. Prozentwert über Klammer: schwere bis mittlere Seborrhö; vertikale Pfeile: keine Seborrhö.

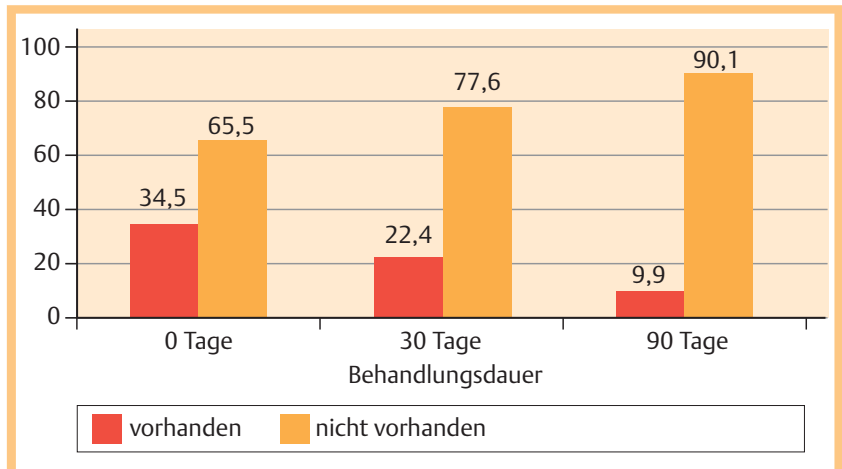

Abb. 8 Verbesserung der seborrhoischen Dermatitis.

Als weiterer Untersuchungsparameter wurde die Präsenz einer seborrhoischen Dermatitis erfasst. Der Anteil der Probanden mit einer seborrhoischen Dermatitis lag bei Beginn der Studie bei $34,5 \%$ und nahm nach 30 Tagen Anwendung der kosmetischen Melatonin-Lösung auf 22,4\% und nach 90 Tagen auf 9,9\% ab. Dementsprechend stieg der Anteil der Patienten, die keine seborrhoische Dermatitis hatten, von $65,5 \%$ auf $77,6 \%$ und $90,1 \%$ an (๑ Abb. 8).

Eine Mehrheit von 74,4\% der Probanden war nach einer Behandlungsdauer von 90 Tagen mit den kosmetischen Resultaten zufrieden, 23,3\% waren mäßig zufrieden und ein kleiner Anteil von 2,3\% war mit dem Ergebnis nicht zufrieden. Gemäß den Einschätzungen der Ärzte und Probanden war die Verträglichkeit der kosmetischen Melatonin-Haarlösung bei einer Mehrheit von 88,0\% bzw. 82,7\% der Anwender gut verträglich, und sowohl die Ärzte als auch die Probanden stuften die Behandlung lediglich in 2,0\% bzw. 3,0\% der Fälle als nicht gut verträglich ein. Nach der Gesamtbeurteilung durch die Ärzte und die Probanden hatte die Melatonin-Lösung in 85,3\% bzw. 80,8\% der Fälle eine größere Wirksamkeit bezüglich der Verbesserung des Haarverlustes als andere vorgängig angewendete Produkte, wohingegen lediglich $0,7 \%$ der Ärzte und $1,0 \%$ der Probanden die Wirksamkeit der kosmetischen Haarlösung gegenüber den vorgängigen Produkten als geringer empfanden.

\section{Diskussion}

$\nabla$

Aus In-vitro-Untersuchungen im Haarorgankulturmodell ist bekannt, dass das Neurohormon Melatonin das Wachstum von humanen Haarfollikeln stimulieren kann [35]. In der vorliegenden Arbeit wurde die Hypothese anhand mehrerer klinischer Studien überprüft, ob die Ergebnisse der In-vitro-Daten in der klinischen Situation in vivo bestätigt werden können.

In der ersten Studie zur Pharmakodynamik und Verträglichkeit fand sich nach topischer Applikation einer 0,0033\%-igen Melatonin-Lösung im Vergleich zur Melatonin-freien Placebo-Lösung ein leicht erhöhter, jedoch nicht signifikant unterschiedlicher Melatonin-Plasmaspiegel [37]. Die kumulative Menge des Melatonin-Metaboliten 6-Hydroxymelatonin, der indirekt die Melatonin-Plasma-Spiegel angibt, war in beiden Gruppen vergleichbar. Neurokognitive Veränderungen und Auswirkungen auf Vitalzeichen konnten nicht beobachtet werden.

In der zweiten Studie bei 30 Patienten im Frühstadium der AGA zeigte Melatonin eine signifikante Reduktion des Haarausfalls nach 30 bzw. 90 Tagen. Zur Bewertung der Studie ist einschränkend festzustellen, dass keine objektiven, bildgestützten Messmethoden, sondern objektive und subjektive Prüfarzt- bzw. Probandenfragebögen verwendet wurden und der beobachtete Effekt nicht durch Placebo kontrolliert wurde. Es ist dennoch festzuhalten, dass ein signifikanter Prä-Post-Effekt beobachtet wurde [38]. Die Verträglichkeit war relativ gut (in 18\% der Fälle Unverträglichkeit) und die Akzeptanz des Produktes mit 90\% hoch.

In der dritten Studie, einer offenen, klinisch-kontrollierten Studie, zeigte eine 6-monatige topische Melatonin-Behandlung mittels TrichoScan, einem objektiven, Auflichtmikroskopie-gestützten, digitalen Evaluationsverfahren $[40,41]$ an 35 Männern mit AGA, bei 54,8\% bzw. 58,1\% der Männer nach 3 bzw. 6 Monaten eine signifikante Zunahme der Haardichte. Diese lag bei $+29 \%$ (Monat 3) bzw. $+41 \%$ (Monat 6) [39]. Eine Verbesserung des Haarausfalls zeigte sich auch in der Prüfarztbeurteilung $27 \%$ bis 48\%; Monat 1 und 3). Eine Stabilisierung des Haarverlustes wurde bei rund $75 \%$ bzw. $50 \%$ beobachtet (Monat 1 bzw. 3 und 6 ). Die kosmetischen Eigenschaften des Haar-Therapeutikums wurden als relativ gut beurteilt und die Verträglichkeit war außer bei einem von 35 Studienteilnehmern ebenfalls gut.

In der vierten Anwenderstudie in vier Coiffeur-Salons an 60 weiblichen und männlichen Probanden mit beginnendem Haarausfall über 90 Tage wurde durch die Coiffeure auf einer 4- bzw. 5-Punkte-Skala im Prä-Post-Vergleich die Haartextur als signifikant gebessert und der Haarverlust als signifikant reduziert beurteilt. Durch einen standardisierten Kämm-Test (60-SekundenHaarzähltest) [43] zeigte sich bei den weiblichen Probanden eine signifikante Abnahme des Haarverlustes, während der Haarverlust bei den Männern konstant aber auf niedrigem Niveau blieb [42]. An Nebenwirkungen traten vorübergehende Rötung, Empfindlichkeit, Juckreiz oder Brennen auf, die nicht zum Studienabbruch führten.

In der fünften, groß angelegten, offenen Multizenterstudie über 3 Monate bei 1891 weiblichen und männlichen Patienten mit AGA im Stadium I und II nahm der Anteil der Patienten mit 2- bis 3fach positivem Pull-Test von 61,6\% zu Beginn auf 7,8\% nach 3 Monaten ab, während der Anteil mit negativem Pull-Test (kein Haarausfall) von $12,2 \%$ auf $61,5 \%$ anstieg [44]. Durch objektive standardisierte Prüfarztfragebögen konnte bei bis zu $66 \%$ eine Abnahme des Haarverlustes und bei bis zu 22,5\% der Probanden nach 3 Monaten erneutes Haarwachstum verzeichnet werden. 
Zusätzlich wurde durch die Prüfärzte eine Abnahme des Anteils der Probanden mit schwerer und mittlerer Seborrhö von 35,7\% auf 5,4\% festgestellt. Damit einhergehend wurde auch eine Abnahme der seborrhoischen Dermatitis von 34,5\% auf 9,9\% beobachtet. Die Verträglichkeit der Melatonin-haltigen Lösung beurteilten $88,0 \%$ der Prüfärzte und $82,7 \%$ der Probanden als gut, während $2 \%$ bzw. $3 \%$ die Behandlung als nicht gut verträglich einstuften.

Die Designs der Studien zur Wirkung von Melatonin auf die AGA bzw. auf Haarausfall müssen unterschiedlich bewertet werden. Während in der ersten Studie von Lorenzi und Caputo (Studie 2) [38] ausschließlich Prüfarzt- und Probandenfragebögen verwendet wurden, die zwar im Falle der Prüfarztbeurteilung eine geschulte, objektiv-klinische Evaluation erlauben, aber dennoch eine höhere Variabilität als objektive Messverfahren aufweisen, wurde in der zweiten Studie von Lorenzi (Studie 3) [39] ein objektives, instrumentengestütztes Messverfahren, die TrichoScanUntersuchung, gewählt und an 35 Männern angewandt. Der intra-individuelle Untersucher-Korrelationskoeffizient der TrichoScan-Methode liegt bei $91 \%$ und der inter-individuelle Untersucher-Korrelationskoeffizient bei $97 \%$ [41]. Dadurch bietet die TrichoScan-Methode eine gute Verlässlichkeit im Prä-Post-Vergleich und ist gut reproduzierbar im Verlauf einer klinischen Studie $[40,41,46]$. Die Zunahme der gemessenen Haarzahl von 123 auf 159 und 173 Haare pro $\mathrm{cm}^{2}$ über 3 bzw. 6 Monate ist als klinisch relevant einzustufen. Die Studie 4 wurde als Anwendungsbeobachtung bei 60 Probanden/innen durchgeführt, wobei sich hinsichtlich Haartextur und Haarverlust signifikante PräPost-Vergleiche feststellen ließen, aber nicht gegen Placebo getestet wurde. Der 60-Sekunden-Haarzähltest ist eine Methode, die vom Probanden selbst durchgeführt wird, jedoch durch die Vorgabe, während 60 Sekunden kontinuierlich zu kämmen, eine gewisse Standardisierung erreicht. Die Methode ist einfach durchzuführen und gibt standardisiert den täglichen Haarverlust wieder [43]. Die Multizenterstudie (Studie 5) ist mit über 1800 Teilnehmern aus 200 Zentren die größte Studie zur Anwendung von Melatonin bei AGA im Frühstadium [44] und übertrifft damit hinsichtlich der Probandenzahl die große Studie zur Anwendung von Finasterid bei der AGA des Mannes mit über 1500 Probanden [47]. Die Methodik des Pull-Testes ist semi-quantitativ und unterliegt einer relativ starken Inter-Untersucher-Variabilität, wobei aber jeder Untersucher üblicherweise für sich selbst eine hohe intra-individuelle Standardisierung erreicht [4,45]. Die Methode liegt hinsichtlich Varianz und Reproduzierbarkeit qualitativ zwar deutlich unter der objektiven Methode TrichoScan, der verblindeten Haarauszählung oder der ÜbersichtsfotografieEvaluation durch ein verblindetes Untersucherpanel, erlaubt aber aufgrund der intra-individuellen Standardisierung durch den einzelnen Prüfarzt eine gewisse Verlässlichkeit der Ergebnisse. Im Fall der vorliegenden Multizenterstudie mit 200 Zentren wurde durch die hohe Zahl der Zentren der Einfluss der Inter-Untersucher-Variabilität auf das Studienergebnis auf ein relativ niedriges Niveau reduziert. Allerdings wurde auch in dieser Studie nicht gegen Placebo getestet, was die Aussagekraft heruntersetzt. Unabhängig davon wurden in der Studie mit Untersuchung der Seborrhö und der Präsenz einer seborrhoischen Dermatitis zwei weitere Parameter erfasst, die einen anderen wichtigen Aspekt der Beeinträchtigung der Patienten darstellen und die auf die Behandlung mit Melatonin gut ansprechen.

Die positiven Effekte von Melatonin auf das Haarwachstum bei Patienten mit AGA bestätigen die Ergebnisse aus einer Pilot-Stu- die zu Melatonin, die als Placebo-kontrollierte, randomisierte, doppel-blinde Studie an 40 Patientinnen mit androgenetischer bzw. diffuser Alopezie durchgeführt wurde [36]. Obwohl der exakte Wirkmechanismus der Wachstumsstimulation von Melatonin auf das menschliche Haar noch nicht eindeutig geklärt ist, gibt es verschiedene Hinweise aus dem Tiermodell oder dem Tier- und humanen Organkulturmodell mit Haarfollikeln in vitro [33]. Vor allem aus dem tierwissenschaftlichen Bereich ist bekannt, dass Melatonin die Woll- und Kaschmirproduktion steigern und die Entwicklung und Zyklus-Regulation des Deckfells, des Fellwechsels und der Fellfarbe modulieren kann $[48,49]$.

Rezeptor-vermittelte Melatonineffekte sind theoretisch möglich, da der Membranrezeptor MT1 sowohl in humanen HaarfollikelKeratinozyten und Fibroblasten der dermalen Haarpapille mittels RT-PCR nachgewiesen [30] als auch in situ in humaner Kopfhaut im mittleren Teil der äußeren und inneren Wurzelscheide des Haarfollikels detektiert worden ist [28]. Auch der Membranrezeptor MT2 ist in einer aberranten Form in humanen Fibroblasten der dermalen Haarpapille nachgewiesen worden [30]. Im Modell mit C57BL/6-Mäusen wurde mittels RT-PCR eine spezifische, haarzyklusabhängige Expression von MT2 in der Haut gezeigt [34]. Funktionell könnte daher dieser Rezeptor einen Einfluss auf den Haarzyklus haben, wenn die Erkenntnisse aus dem Maus-Modell mit auf den Menschen übertragen werden können. Am humanen Haarfollikel konnte MT2 bisher nur schwach in der inneren Wurzelscheide mittels Immunhistochemie detektiert werden [28]. Der MT3-Rezeptor bzw. NQO2 ist zwar in humanen Einzelzellen der Haut (Keratinozyten, Melanozyten, Fibroblasten), bisher jedoch noch nicht in Einzelzellen des Haarfollikels noch im Haarfollikel in situ nachgewiesen worden [27]. MT3/ NQO2 könnte eine Rolle in der Prävention von oxidativem Stress in der HF-Katagen-Regression oder bei der Haaralterung, die durch oxidativen Stress bedingt ist, [12] spielen. Der nukleäre Melatoninrezeptor ROR $\alpha$ übt in der Maus eine biologische Funktion auf das Haarwachstum aus, da in ROR $\alpha$-knock-out-Mäusen das Fell deutlich gelichtet ist [33]. In C57BL/6-Mäusen konnte auch eine haarzyklusabhängige Regulation des nukleären Rezeptors in der inneren und äußeren Wurzelscheide festgestellt werden [34]. Die Lokalisation aller bisher detektierten Melatoninrezeptoren wurde in der Wurzelscheide des Haares identifiziert, die neben der mechanischen Stabilisation des Haarschaftes regulatorische Funktionen auf das Haarwachstum ausübt. Daher kann vermutet werden, dass die wachstumsfördernden Eigenschaften von Melatonin zumindest teilweise über dessen Rezeptoren in der Haarwurzelscheide reguliert werden.

Die Wirkung von Melatonin auf das Haarwachstum kann auch durch Interaktion mit Androgenen und Östrogenen sowie deren Rezeptoren vermittelt sein. Hinweis dafür ist die antiandrogene Wirkung von Melatonin an benignen Prostatazellen [50]. Während an Prostatazellen die antiandrogene Wirkung mit Proliferationshemmung einhergeht, bewirkt eine antiandrogene Wirkung am Haarfollikel eine Verlängerung des Haarzyklus und Verminderung der Miniaturisierung [2]. Humane benigne Prostata-Zellen und humane Haut exprimieren funktionell aktive Melatonin-Rezeptoren (MT1) [30,51], sodass eine Melatonin-Rezeptorvermittelte antiandrogene Wirkung auch in der Haut und am Haarfollikel angenommen werden kann.

Melatonin zeigte auch modulierende Wirkungen auf die für die Östrogenbildung notwendige Aromataseaktivität und Genexpression, allerdings an Mamma-Ca-Zelllinien [52]. An murinen Haarfollikeln reguliert Melatonin haarzyklusabhängig die $\alpha$ - 
Östrogen-Rezeptor-Genexpression [34]. Die Relevanz für den humanen Haarfollikel muss in funktionellen Studien noch geklärt werden.

Zusammenfassend kann festgestellt werden, dass in mehreren Studien mit unterschiedlichen Test-Designs überwiegend positive Effekte durch die topische Anwendung einer kosmetischen Melatonin-Lösung bei Frauen und Männern mit AGA in frühen Stadien (Hamilton bzw. Ludwig I und II) mit teilweise deutlichem Rückgang des Haarverlustes oder auch erneutem Haarwachstum beobachtet werden konnten. Obwohl der Wirkmechanismus noch nicht detailliert geklärt ist, erscheint eine über MelatoninRezeptoren vermittelte antiandrogene Wirkung und/oder eine antioxidative Wirkung sehr wahrscheinlich zugrunde zu liegen. Da sich die Anwendung der Melatonin-Lösung in insgesamt 5 Studien als sehr gut verträglich erwiesen hat und im Prä-PostVergleich positive Effekte auf das Haarwachstum beobachtet werden konnten, stellt die kosmetische Melatonin-Lösung eine mögliche Option zur Verzögerung der Haaralterung, von Haarausfall allgemein und zur ergänzenden Behandlung der AGA bei Männern und Frauen im Frühstadium dar.

\section{Interessenkonflikt}

Tobias W. Fischer ist für folgende Firmen als wissenschaftlicher Berater zum Thema Melatonin und Haarwachstum tätig: ASATONA AG, Zug/Schweiz; Hans Karrer GmbH, Augsburg. Er war als Sub-Investigator für eine Studie tätig, die an der Klinik für Dermatologie und dermatologische Allergologie der Friedrich-Schiller-Universität Jena, die im Auftrag der Fa. ASATONA AG durchgeführt wurde.

Ralph M. Trüeb ist für die Fa. ASATONA AG, Zug/Schweiz, als Consultant tätig.

Gabriella Hänggi ist als Consultant Scientific Communication für die Fa. ASATONA AG, Zug/Schweiz, tätig.

Marcello Innocenti war am Instituto Dermatologico Europeo (IDE), Mailand/Italien, mit der Auswertung der Studien MELCOS-AS03 und MEL-COS-AS05 betraut. Er erhielt keine Kompensation von ASATONA AG. ASATONA AG sponsorte die Studie, die am IDE durchgeführt wurde.

Peter Elsner war für die Fa. ASATONA AG, Zug/Schweiz, als Referent und Investigator tätig.

\section{Abstract}

\section{Topical Melatonin for Treatment of Androgenetic Alopecia}

On the search for new substances against androgenetic alopecia, melatonin, a strong antioxidant and growth modulator was identified as a promising candidate from in vitro and in vivo studies. Five clinical studies showed positive effects of a topical melatonin solution in the treatment of AGA in men and women while showing good tolerability: (1) Pharmacodynamics under once daily topical application in the evening showed no significant influence on endogenous melatonin plasma levels. (2) A use test with 30 men and women showed by evaluation of investigators and patients questionnaires a significant reduction of severeness of alopecia after 30 and 90 days ( $p<0.001)$. (3) Using a digital software-supported epiluminescence-technique (TrichoScan) in 35 men with AGA, after 3 and 6 months in $54.8 \%$ to $58.1 \%$ of the patients a significant increase of hair density of $29 \%$ and $41 \%$, respectively was measured (M0: 123/ $\mathrm{cm}^{2}$; M3: 159/ $\mathrm{cm}^{2}$; M6: $\left.173 / \mathrm{cm}^{2}\right)(\mathrm{p}<0.001)$. (4) In 60 men and women with hair loss, a significant reduction of hair loss was observed in women, while hair loss in men was kept on a constant level $(\mathrm{p}<0.001)$. (5) In a large 3-months multi-center study with more than 1800 volunteers in 200 centers, the percentage of patients with 2- to 3-fold positive hair pull test decreased from $61.6 \%$ to $7.8 \%$, while the percentage of patients with negative hair pull test increased from $12.2 . \%$ to $61.5 \%(p<0.001)$. Additionally, a decrease of seborrhea and seborrhoic dermatitis of the scalp skin was observed. Since safety and tolerability in all studies was good, the topical application of a cosmetic melatonin solution can be considered as a meaningful treatment option or complementary treatment of androgenetic alopecia.

\section{Literatur}

1 Fischer TW, Schmidt S, Strauss B, Elsner P. Hairdex - ein Instrument zur Untersuchung der krankheitsbezogenen Lebensqualität bei Patienten mit Haarerkrankungen. Hautarzt 2001; 52: 219-227

2 Trueb RM. Molecular mechanisms of androgenetic alopecia. Exp Gerontol 2002; 37: 981 - 990

3 Hoffmann R, Happle R. Current understanding of androgenetic alopecia. Part I: etiopathogenesis. Eur J Dermatol 2000; 10: 319- 327

4 Fischer TW. Alopezien - Diagnostisches und therapeutisches Management. Akt Dermatol 2008; 34: 209-225

5 Hamilton JB. Patterned loss of hair in man; types and incidence. Ann $\mathrm{N}$ Y Acad Sci 1951; 53: $708-728$

6 Norwood OT. Male-pattern baldness. Classification and incidence. South Med J 1975; 68: 1359-1370

7 Ludwig E. Classification of the types of androgenetic alopecia (common baldness) occurring in the female sex. Br J Dermatol 1977; 97: $247-$ 254

8 Ramot Y, Czarnowicki T, Zlotogorski A. Finasteride induced Gynecomastia: Case report and Review of the Literature. Int J Trichology 2009; 1: 27-29

9 Dawber RP, Rundegren J. Hypertrichosis in females applying minoxidil topical solution and in normal controls. J Eur Acad Dermatol Venereol 2003; 17: $271-275$

10 Trueb RM. Das Haar im Alter. Haut 2008; 4: 152-155

11 Mahé YF, Michelet JF, Billoni N et al. Androgenetic alopecia and microinflammation. Int J Dermatol 2000; 39: 576-584

12 Trüeb RM. Oxidative stress in Ageing of Hair. Int J Trichol 2009; 9: 6-14

13 Camacho F, Moreno JC, Garcia-Hernandez MJ. Telogen alopecia from UV rays. Arch Dermatol 1996; 132: 1398 - 1399

$14 \mathrm{Lu}$ ZF, Fischer TW, Hasse S et al. The human hair follicle as a model for exploring the effects of ultraviolet radiation in a complex tissue interaction system in situ. J Invest Dermatol 2009; 129: 1790 - 1804

15 Trüeb RM. Association between smoking and hair loss: another opportunity for health education against smoking? Dermatology 2003; 206: $189-191$

$16 \mathrm{Su} \mathrm{LH}$, Chen TH. Association of androgenetic alopecia with smoking and its prevalence among Asian men: a community-based survey. Arch Dermatol 2007; 143: 1401-1406

17 Srogi $K$. Mercury content of hair in different populations relative to fish consumption. Rev Environ Contam Toxicol 2007; 189: 107-130

18 Bahta AW, Farjo B, Philpott MP. Premature senescence of balding dermal papilla cells in vitro is associated with p16INK4a expression. J Invest Dermatol 2008; 128: 1088 - 1094

19 Tan DX, Chen LD, Poeggeler B et al. Melatonin: a potent, endogenous hydroxyl radical scavenger. Endocr J 1993; 1: 57-60

20 Lerner AB, Case JD, Takahashi Y. Isolation of melatonin, a pineal factor that lightens melanocytes. J Am Chem Soc 1958; 80: 2587

21 Arendt J. Melatonin. Clin Endocrinol (Oxf) 1988; 29: 205-229

22 Lerchl A, Schlatt S. Influence of photoperiod on pineal melatonin synthesis, fur color, body weight, and reproductive function in the female Djungarian hamster, Phodopus sungorus. Neuroendocrinology 1993; 57: $359-364$

23 Karasek M, Reiter RJ. Melatonin and aging. Neuroendocrinol Lett 2002; 23 (Suppl 1): 14-16

24 Fischer TW, Scholz G, Knoll B et al. Melatonin reduces UV-induced reactive oxygen species in a dose-dependent manner in IL-3-stimulated leukocytes. J Pineal Res 2001; 31: 39-45 
25 Fischer TW, Zmijewski MA, Wortsman J, Slominski A. Melatonin maintains mitochondrial membrane potential and attenuates activation of initiator (casp-9) and effector caspases (casp-3/casp-7) and PARP in UVR-exposed HaCaT keratinocytes. J Pineal Res 2008; 44: 397-407

26 Reiter RJ, Tan DX, Poeggeler B et al. Melatonin as a free radical scavenger: implications for aging and age-related diseases. Ann N Y Acad Sci 1994; 719: $1-12$

27 Slominski A, Fischer TW, Zmijewski MA et al. On the role of melatonin in skin physiology and pathology. Endocrine 2005; 27: 137-148

28 Slominski A, Wortsman J, Tobin DJ. The cutaneous serotoninergic/melatoninergic system: securing a place under the sun. FASEB J 2005; 19 : $176-194$

29 Slominski A, Pisarchik A, Semak I et al. Serotoninergic and melatoninergic systems are fully expressed in human skin. FASEB J 2002; 16 : $896-898$

30 Slominski A, Pisarchik A, Zbytek B et al. Functional activity of serotoninergic and melatoninergic systems expressed in the skin. J Cell Physiol 2003; 196: $144-153$

31 Fischer TW, Sweatman TW, Semak I et al. Constitutive and UV-induced metabolism of melatonin in keratinocytes and cell-free systems. FASEB J 2006; 20: $1564-1566$

32 Fischer TW, Slominski A, Tobin DJ, Paus R. Melatonin and the hair follicle. J Pineal Res 2008; 44: 1 - 15

33 Fischer TW. The influence of melatonin on hair physiology. Hautarzt 2009; 60: 962 - 972

34 Kobayashi H, Kromminga A, Dunlop TW et al. A role of melatonin in neuroectodermal-mesodermal interactions: the hair follicle synthesizes melatonin and expresses functional melatonin receptors. FASEB J 2005; 19: 1710 - 1712

35 Fischer TW, Fischer A, Knöll B et al. Melatonin in low doses enhances in vitro human hair follicle proliferation and inhibits hair growth in high doses. Arch Derm Res 2000; 292: 147

36 Fischer TW, Burmeister G, Schmidt HW, Elsner P. Melatonin increases anagen hair rate in women with androgenetic alopecia or diffuse alopecia: results of a pilot randomized controlled trial. $\mathrm{Br} \mathrm{J}$ Dermatol 2004; $150: 341-345$

37 Macher JP. Pharmacokinetics and clinical and biological tolerability of repeated topical application of a melatonin-containing cosmetic hair solution in healthy female volunteers. A double-blind, placebo-controlled, cross-over design study. Clinical Study Report. MEL-COS-1. Data on file. Asatona AG, Switzerland

38 Lorenzi S, Caputo R. Melatonin cosmetic hair solution: Open study of the efficacy and the safety on hair loss (telogen) control and hair growth (anagen) stimulation. MEL-COS-AS01. Data on file. Asatona AG, Switzerland

39 Lorenzi S, Barbareschi M, Caputo R. Efficacy and safety of a melatonincontaining cosmetic hair solution in the treatment of early stages of male androgenic alopecia. Open study with Trichoscan evaluation. Report/Protocol. MEL-COS-AS03. Data on file. Asatona AG, Switzerland

40 Gassmueller J, Rowold E, Frase T, Hughes-Formella B. Validation of TrichoScan technology as a fully-automated tool for evaluation of hair growth parameters. Eur J Dermatol 2009; 19: 224-231

41 Hoffmann R. TrichoScan: combining epiluminescence microscopy with digital image analysis for the measurement of hair growth in vivo. Eur J Dermatol 2001; 11: $362-368$

42 Schmid HW. Use Test with of a melatonin-containing cosmetic hair solution to determine the change of the appearance and texture of thinning and fine hair following the application. Statistical Report. MEL-COS-AS04. Data on file. Asatona AG, Switzerland

43 Miller JJ, Fang S. Results of 60-second timed hair counts in women between the ages of 20-60 years. J Am Acad Dermatol 2004; 50: 93

44 Innocenti $M$, Barbareschi $M$. Open-label, non comparative, multicenter clinical study on efficacy and safety of a melatonin-containing cosmetic hair solution in the treatment of hair loss (telogen) and in the stimulation of hair re-growth (anagen). Report. MEL-COS-AS05. Data on file. Asatona AG, Switzerland

45 Hillmann K, Blume-Peytavi U. Diagnosis of hair disorders. Semin Cutan Med Surg 2009; 28: $33-38$

46 Hoffmann R. Trichoscan: what is new? Dermatology 2005; 211: 54-62

47 Kaufman KD, Olsen EA, Whiting D et al. Finasteride in the treatment of men with androgenetic alopecia. Finasteride male pattern hair loss study group. J Am Acad Dermatol 1998; 39: 578-589

48 Ibraheem M, Galbraith H, Scaife J, Ewen S. Growth of secondary hair follicles of the Cashmere goat in vitro and their response to prolactin and melatonin. J Anat 1994; 185 (Pt 1): 135-142

49 Nixon AJ, Ashby MG, Saywell DP, Pearson AJ. Seasonal fiber growth cycles of ferrets (Mustela putorius furo) and long-term effects of melatonin treatment. J Exp Zool 1995; 272: 435-445

50 Alonso R, Prieto L, Hernandez C, Mas M. Antiandrogenic effects of the pineal gland and melatonin in castrated and intact prepubertal male rats. J Endocrinol 1978; 79: 77-83

51 Gilad E, Matzkin H, Zisapel N. Interplay between sex steroids and melatonin in regulation of human benign prostate epithelial cell growth. J Clin Endocrinol Metab 1997; 82: 2535-2541

52 Cos S, Gonzalez A, Martinez-Campa Cet al. Estrogen-signaling pathway: a link between breast cancer and melatonin oncostatic actions. Cancer Detect Prev 2006; 30: 118-128 